\title{
A low-cost do-it-yourself microscope kit for hands-on science education
}

\section{Zoe Grier, Maria Francesca Soddu, Naomi Kenyatta, Sharlene Agyei Odame, Joshua Sanders, et al.}

Zoe Grier, Maria Francesca Soddu, Naomi Kenyatta, Sharlene Agyei Odame, Joshua Sanders, Latasha Wright, Francesca Anselmi, "A low-cost do-ityourself microscope kit for hands-on science education," Proc. SPIE 10741, Optics Education and Outreach V, 107410K (14 September 2018); doi: $10.1117 / 12.2320655$

EDIE Event: SPIE Optical Engineering + Applications, 2018, San Diego, California, United States 


\title{
A low-cost do-it-yourself microscope kit for hands-on science education
}

\author{
Zoe Grier $^{1 *}$, Maria Francesca Soddu ${ }^{1,2 *}$, Naomi Kenyatta ${ }^{1}$, Sharlene Agyei Odame ${ }^{1}$, Joshua \\ Sanders $^{3}$, Latasha Wright ${ }^{1 \#}$ and Francesca Anselmi ${ }^{1 \# \$}$ \\ *\# equal contribution \\ ${ }^{1}$ BioBus, Inc., USA \\ ${ }^{2}$ Columbia University's Zuckerman Institute \\ ${ }^{3}$ Sanworks, LLC, USA \\ \$francesca@biobus.org
}

\begin{abstract}
Microscopes play a central role in hands-on science exploration and communication. All too often, however, students do not have a good understanding of the optical principles that govern microscopy and lack access to instruments that could help them build that understanding. Here we present an open-source Do-It-Yourself (DIY) microscope kit developed by scientists and students at BioBus, a nonprofit organization for science outreach and education based in New York City (www.biobus.org). The DIY microscope uses 3D printing to make highly adaptable optical technology readily available to educators at a low cost. Its modular configuration makes it the perfect tool to teach optical design to students of all ages. At BioBus, Inc., over 230 school-age students and teachers learned basic optics and microscope building with the DIY microscope at our community and mobile laboratories.

We further present examples of how the DIY microscope was used as a platform for student-driven projects, expanding the original design to include advanced optical features such as fluorescence and infrared imaging. The images, acquired with a low cost camera, were comparable in quality to those taken using professional grade laboratory microscopes. The use of the DIY microscope is not limited to applications in physical sciences, but can also be used as an interdisciplinary teaching tool. As an example, we showed how it was configured into a functional model of the eye, to explain the physics of vision and the pathophysiology of eye disorders, such as far and short-sightedness, and age-related macular degeneration.
\end{abstract}

Keywords: microscope, do-it-yourself, education, open source, 3D-printed, mobile laboratory, community laboratory, outreach

\section{INTRODUCTION}

Microscopes are among the most versatile and powerful scientific instruments. Their introduction in the 16th century led to an explosion of scientific progress, including the discovery of cells as the fundamental unit of life, and the associated realization that the world is full of microorganisms too small to be seen by the naked eye. Since then, microscopes have become an essential tool for research in every branch of science ${ }^{1}$. Conventional light microscopy has been complemented by the development of techniques with ever finer resolution, including electron ${ }^{2}$ and $\mathrm{x}$-ray ${ }^{2,3}$ microscopy. In more recent years, scientists have developed new approaches to light microscopy that have greatly extended its reach, as exemplified by fluorescence confocal microscopy ${ }^{4}$ and two-photon laser scanning microscopy ${ }^{5}$, which allow mapping structures within living cells and even entire organisms.

In the framework of science outreach and education, microscopes can play a different but equally important role. By providing an immediate visual experience of the inner working of life and matter, microscopes can create a natural gateway to science for students of all ages. Scientists at BioBus Inc. and other similar organizations around the world (www.mobilelabcoalition.com) have been testing this hypothesis by equipping mobile and community laboratories with research-grade microscopes, thus making these powerful instruments accessible to students, teachers and the general

Optics Education and Outreach V, edited by G. Groot Gregory, Proc. of SPIE Vol. 10741, 107410K

(C) 2018 SPIE · CCC code: 0277-786X/18/\$18 · doi: 10.1117/12.2320655 
public. Since BioBus was founded in 2008, more than 250,000 students from over 600 schools, primarily in the New York City area, have experienced hands-on science exploration at BioBus laboratories, using microscopes typically available only to research scientists and graduate students (www.biobus.org).

Paradoxically, however, these same microscopes can also feel like the embodiment of everything that seems difficult and out-of-reach in science in the minds of teenagers and young adults. Enclosed in their industrially-designed cases, microscopes are often presented to students as inscrutable black boxes whose design principles can only be understood with advanced knowledge that is out of their reach. This impression is reinforced by the high price of research-grade microscopes that prevents them from being widely accessible in schools. As a result, students' access to these instruments is limited to exceptional circumstances such as a BioBus mobile laboratory visit, currently available to a maximum of 60,000 students per year, while there are over one million public school students in NYC alone. Furthermore, economic constraints and the scarcity of professional development opportunities for teachers make it almost impossible for students to experiment with microscope design and building. Such hands-on experiences are invaluable because they provide not only factual knowledge of the underlying physics, but also a sense of agency in the world, teaching students that they have the ability to internalize complex concepts and use their understanding to bring their ideas to a physical realization ${ }^{6}$. Moreover, advanced optical techniques, such as fluorescence microscopy, remain out of reach for the average school-aged student, despite playing a fundamental role in modern scientific research. This situation especially impacts historically underserved communities, further hindering the access of minority and low-income students to scientific careers ${ }^{7,8}$.

To overcome these barriers, two years ago BioBus started the Do-It-Yourself (DIY) microscope project, addressing the need for affordable and flexible microscopes. We decided to capitalize on the rapid increase in the availability of 3D printers in makerspaces around the country and develop a modular open-source optical kit made with 3D printed components. The project's joint goals are to make microscopy affordable for science classrooms around the country, and to develop a modular platform for teaching microscope design and building. With less than $\$ 200$ in production cost, the BioBus DIY optical kit is affordable enough to be deployed in economically disadvantaged communities. Its open design and construction provide students with a hands-on experience of optics that supports the classroom learning experience. Versions of this instrument, moreover, are functional enough to provide the basis for advanced microscopy techniques. This is demonstrated here by the development of two student-driven projects, adapting the DIY optical kit for fluorescence and infrared microscopy. Another student-led project shows how the DIY optical kit can be used as a flexible platform for interdisciplinary learning, by creating a functional model of the human eye and eye disorders.

\section{MATERIALS AND METHODS}

\subsection{The basic kit}

The lens and diffuser mounts, LED holder, sample and screen holders, camera finder and rail-bases for the DIY optical kit were custom designed for 3D printing by Joshua Sanders (https://sanworks.io/), using Sketchup Make software. Parts were prepared for 3D printing using Ultimaker Cura software, then printed with polylactic acid (PLA) plastic filament, using an Ultimaker 2+ 3D printer with a $0.4 \mathrm{~mm}$ nozzle. Speed and resolution parameters were adjusted to guarantee high print quality. For structural support with reproducible alignment, we used a $25 \mathrm{~mm}$ square extruded aluminum T-slot rail (AluFab). The cost of the kit was minimized by purchasing all lenses, except for the objective, from Anchor Optics as optical surplus material (collimator and 4X tube lens: plano-convex lens, focal length - FL $=70 \mathrm{~mm}$; condenser and eyepiece: plano-convex lens, $\mathrm{FL}=30 \mathrm{~mm}$; $20 \mathrm{X}$ tube lens: plano-convex lens, $\mathrm{FL}=400 \mathrm{~mm}$ ). The use of 3D printed parts allowed us to customize each optical mount to fit the diameters of the particular lenses in each surplus lot. As an objective, we used a plastic aspherical lens from Edmund Optics (part number 66-006; FL = 17.5; NA = 0.71) to ensure better imaging quality. The objective mount was designed as two components connected by a plastic thumb screw (DigiKey, part number 36-2502-ND), to allow for fine focusing. A 120 grit ground glass diffuser (Edmund Optics, part number 83-419) was inserted immediately after the light source to homogenize the illumination. All mounts could be secured with a thumb screw to movable bases inserted into the rail, to ensure maximal flexibility and modularity. The plastic thumb screws (part number 36-2501-ND) were obtained from DigiKey, and the corresponding metal nuts from Radio Shack (6403018). The illumination source was assembled from custom and commercial parts and included: a 
white LED (Digikey, part number: CXA1304-0000-000C00C250F-ND), a LED holder assembly and screws (Digikey, part number WM10644-ND, 36-9902-ND), a heat sink (Digikey, part number AE10837-ND), a LED driver (Sparkfun, part number: COM-13716), a custom board designed by J. Sanders, a DC power jack (Sparkfun, part number: PRT-00119), and a 12V DC adapter (Digikey, part number: 237-1455-ND). The modular nature of the kit allows it to be aligned in multiple configurations, including a finite conjugate and an infinite conjugate compound microscope (video at: https://youtu.be/Q1TGvv6tvhI; credit Michael Seto / Michael Seto Photography). Images can either be projected onto a paper screen or recorded on a phone camera. The maximal magnification $(\sim 200 \mathrm{X})$ was obtained in the infinite conjugate configuration using a phone camera. In this case, the image produced by the objective and tube lens (20X) was further magnified by the eyepiece and built-in camera lens $(\sim 10 \mathrm{X})$.

\subsection{Add-ons: fluorescence DIY microscope}

To build a DIY trans-fluorescence microscope, the DIY optical kit was initially aligned as an infinite conjugate compound microscope. Then the white LED light source was substituted with a blue LED $(440-460 \mathrm{~nm}$, Nightsea Stereo Microscope Fluorescence adapter, supplemented with a 450-490 nm bandpass excitation filter - Nikon), and a green bandpass emission filter (520 nm longpass filter - Nikon) was inserted just before the camera. Images were recorded with a Samsung Galaxy S6 camera, or with the Raspberry Pi camera module V2, connected to a Raspberry Pi 3 model B single-board computer. Fluorescent samples of fruit fly larvae expressing GFP in class one neurons (221Gal4-CD8-GFP) were kindly provided by Natalie Kolba (Wesley Grueber's laboratory, Columbia University).

\subsection{Add-ons: IR microscope}

To adapt the DIY microscope for infrared (IR) illumination, the broad-spectrum white LED light source was substituted with a narrow spectrum IR $850 \mathrm{~nm}$ LED light (Sparkfun, part number: COM-09854). Images were acquired with a Raspberry Pi Noir camera, connected to a Raspberry Pi 3 model B single-board computer. The microscope was aligned in an infinite conjugate configuration, as described above.

\subsection{DIY eye model.}

The DIY eye model was assembled from the basic kit components. The object (a "smiley face" drawn onto a microscopy slide) was placed into the sample holder and illuminated by the white LED. The following optical components were used: plano convex lens $\mathrm{FL}=70 \mathrm{~mm}$ (cornea); plano convex lens $\mathrm{FL}=70 \mathrm{~mm}$ or $400 \mathrm{~mm}$ (crystalline lens), plano convex lens $\mathrm{FL}=70 \mathrm{~mm}$ (convex correction glasses). A white screen was used as a proxy of the retina. An additional concave lens $(\mathrm{FL}=-45 \mathrm{~mm})$ was purchased from Anchor to mimic concave correction glasses. The implantable miniature telescope was modeled using a convex lens $(\mathrm{FL}=70 \mathrm{~mm})$ and concave lens $(\mathrm{FL}=-45 \mathrm{~mm})$ aligned to form a Galilean telescope.

\subsection{Image processing and data analysis}

All images were processed in ImageJ (https://imagej.nih.gov/ij/). Data from the surveys was analyzed using a spreadsheet application (Google Sheets).

\section{RESULTS}

\subsection{Optical design of the DIY microscope kit}

We designed an optical kit that can be 3D printed and includes all the components of a traditional compound light microscope (light source, diffuser, condenser and collector lenses, sample holder, objective, tube lens, camera holder and eyepiece - Figure 1A-C). Every component can be independently arranged on a rail by securing it to a movable base with a plastic thumb screw (Figure 1C). The modular nature of the kit was chosen to allow students to come up with their own microscope designs, increasing the pedagogical value of the project. All the structural components of the kit 
were custom-designed using 3D printer software (Figure 1B), as described in Methods. To further restrict the cost, the lenses were obtained from surplus optical suppliers, and the design of the lens mounts was adapted to non-standard optical sizes. As an objective, we used a plastic aspherical lens with 0.71 numerical aperture. The objective mount includes a fine focusing mechanism, composed of two adjustable pieces connected by a thumb screw (Figure 1C). Images of the sample can be projected on a screen (Figure 1A, D, F), observed through an eyepiece or acquired with a phone camera (Figure 1E, G). All of the 3D printing design files are open source and can be obtained by contacting the authors.

Figure 1A shows a possible configuration of the optical kit, aligned into a simple finite conjugate microscope. The image of a brain cortical slice was projected into a paper screen: the individual Golgi-stained cells were visible as black dots. Figure 1E shows the same brain preparation imaged through a phone camera, with the kit aligned in an infinite conjugate configuration ( 200X magnification, split between objective, tube lens and eyepiece): the individual brain cells are visible, as well as smaller subcellular structures such as dendrites (typically 1-10 $\mu \mathrm{m}$ ). The same microscope design was used to image cells in the leaves of the aquatic plant Elodea (Figure 1G) and the small crustacean Daphnia magna (Figure 1D - screen projection - and Video 1 - phone camera). In the latter, microscopic anatomical details of the body (eggs, heart, compound eye and filtering apparatus) are clearly distinguishable.
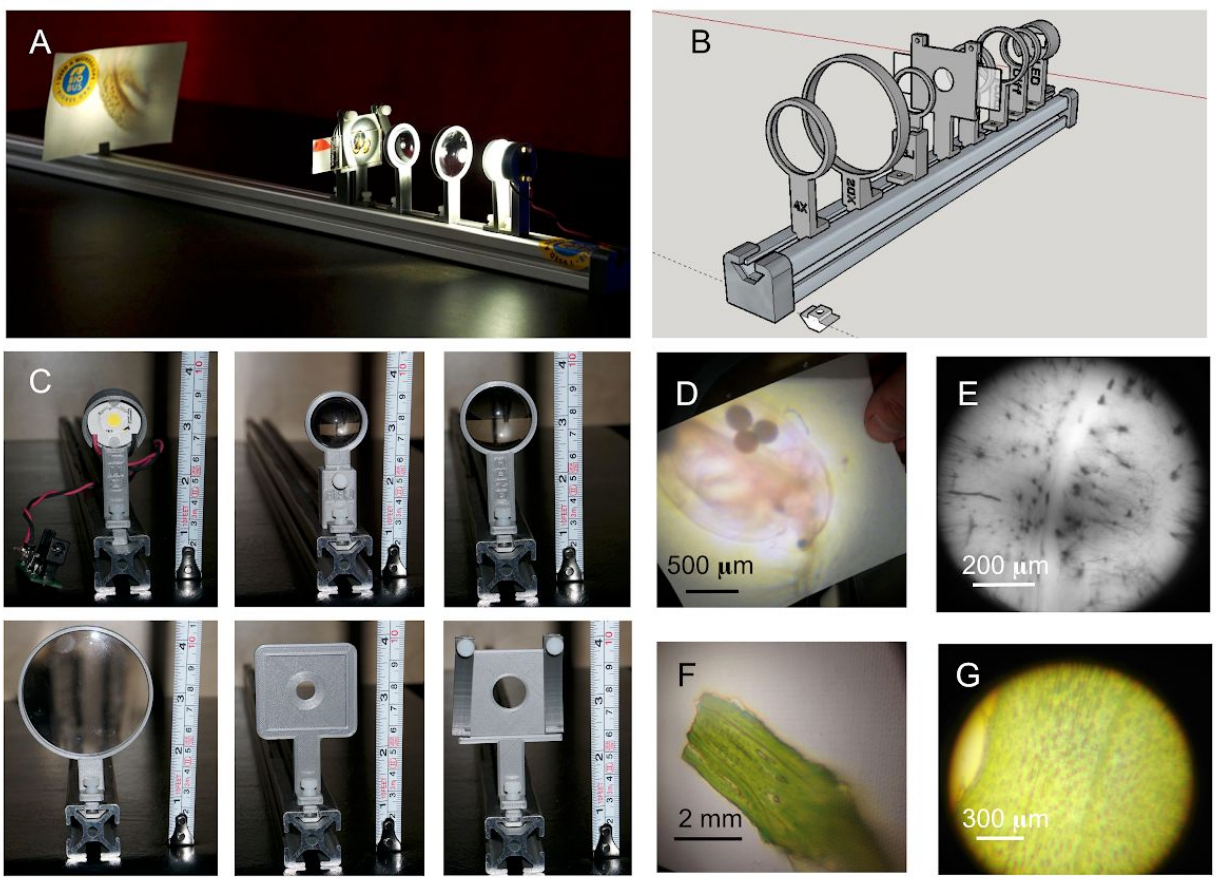

Figure 1: The BioBus DIY microscope kit. A) BioBus DIY, 3D printed optical kit: the lenses are aligned to form a finite conjugate microscope, imaging a Golgi-stained brain slice; B) design files for 3D printing the different components of the DIY optical kit; C) individual kit components: (top left to bottom right) LED light source, objective with fine focusing mechanism, condenser lens, 20X tube lens, camera finder, sample holder; D-G) examples of images taken with the DIY microscope: the freshwater crustacean Daphnia magna (D), Golgi stained neurons in a mouse brain slice (E), a grass root (F); a leaf section of the aquatic plant Elodea (G).

\subsection{Science outreach programs}

Since the beginning of the BioBus DIY microscope project in 2016, over 230 students and teachers participated in programs featuring the DIY optical kit at BioBus and at our partner organizations (Figure 2). We developed two sets of guided-inquiry curricula, using the DIY optical kit to explore the fundamentals of optics and microscopy, as well as the physics of vision and the human eye. Additionally, we pioneered an open-inquiry approach, where students as young as 
8 years old were encouraged to come up with their own, creative microscope designs without following any specific set of instructions (Figure 2B). The latter produced the most surprising results, as our elementary school students conceived and realized original optical designs with minimal supervision, including a microscope able to image two samples in alternative optical planes (Matthew Diaz and Sawyer King, 9 years old; video at https://youtu.be/0wm1Isp-Vro).
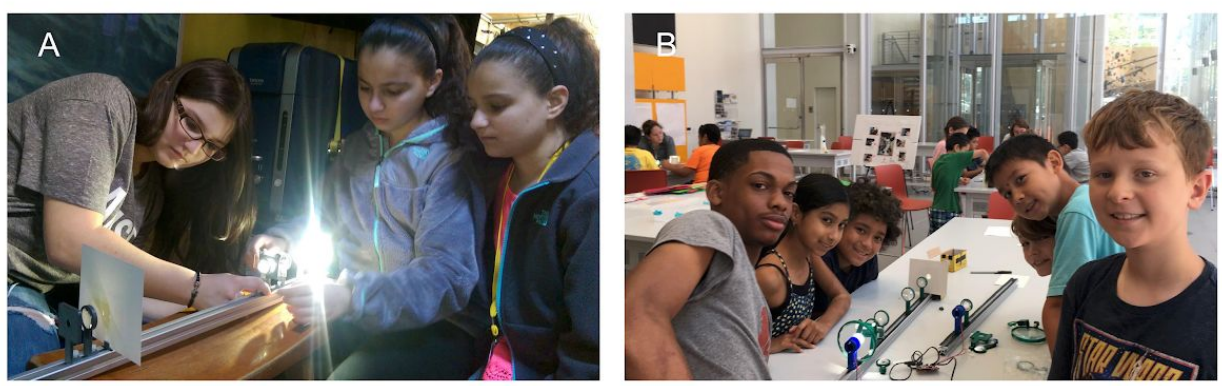

Figure 2: BioBus and BioBase programs. (A) Students using the DIY microscope at a public event on the BioBus mobile laboratory; (B) Elementary school students engineering their own DIY microscope at BioBus community laboratory.

The DIY microscope lesson plans were tested with multiple age groups (elementary, middle and high school students), and in different informal educational settings, including week-long outside of school time programs (summer camps and after school), one-day workshops, 45-minute lessons on BioBus mobile laboratories, and public events with large public attendance (i.e. New York Maker Faire, World Science Festival). Additionally, we conducted professional development courses for teachers of mixed grades. For some of these programs, formal feedback was collected by BioBus and by our partner organizations. The results are reported in Figure 3. Students and parents/guardians (when present at the program) consistently found the DIY microscope approach to optics interesting (or worth recommending to other students), and useful to better understand how microscopes work (Figure 3A, B). High school students from the Washington Heights Expeditionary Learning School (WHEELS) in Manhattan also expressed interest in having the DIY optical kit available at their school (Figure 3A). This sentiment was echoed by teachers from our partner program, Math for America (MfA, https://www.mathforamerica.org/). MfA recruits top public school mathematics and science teaches, creating a community of outstanding educators who share best teaching practices. It also partners with scientists and informal educators to provide professional development opportunities for their fellows. During two consecutive years, MfA teachers from mixed grades (elementary to high school) and disciplines participated in a professional development mini-course on microscopes, where the DIY optical kit figured as the central piece. They reported that the course broadened their professional interest. Moreover, a majority of teachers were interested in sharing learnings from the course with colleagues and/or continuing to work on ideas from the course at their schools (data kindly provided by John Russell, Math for America, Figure 3C).

\subsection{Advanced student projects}

Besides being used as a pedagogic tool for grade students, the DIY optical kit was the starting point for the development of advanced, student-driven projects conducted by BioBus high school and college interns. The following paragraphs describe three of those projects. The first two aimed at increasing the optical power of the DIY kit, converting it into a fluorescent microscope, or using infrared illumination to eliminate distortions caused by chromatic aberration. The third project converted the DIY optical kit into a functional model of the human eye, and used it to illustrate the mechanisms of common optical defects and disorders. 

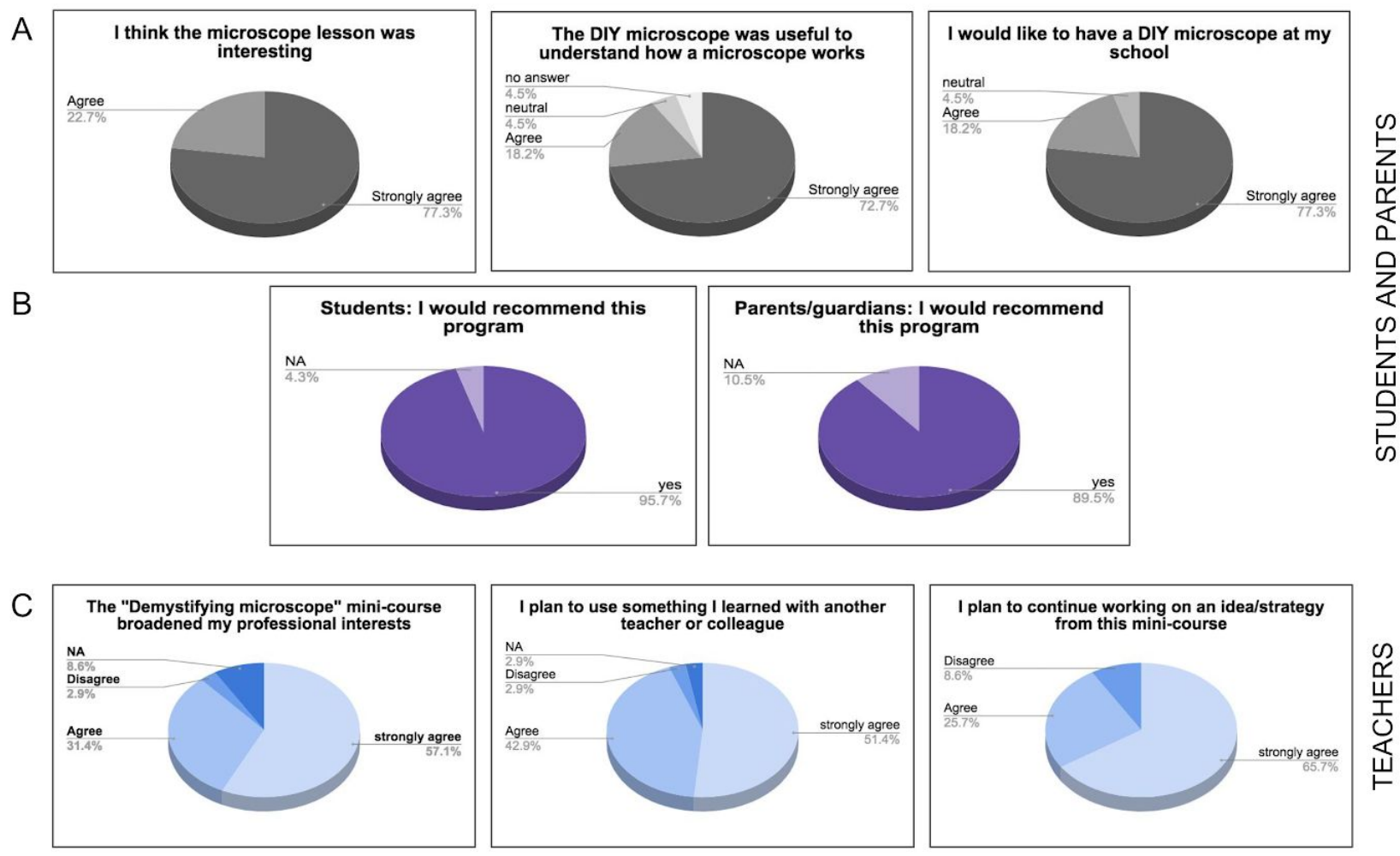

Figure 3: Feedback on DIY microscope programs A-grey) feedback collected from students $(\mathrm{n}=22)$ at the Washington Heights Expeditionary Learning School in NYC: students boarded the BioBus mobile laboratory and designed their own microscope with the DIY optical kit, as well as exploring model organisms using the research quality microscopes in the laboratory; B-purple) feedback collected from students $(n=23)$ and parents/guardians $(n=19)$ during a physics and optics course realized in collaboration between BioBus Inc., New York University - Material Research Science and Engineering Center and the Johns Hopkins Center for Talented Youth: students built their own microscope using the DIY optical kit, guided by scientists from BioBus and NYU (data kindly provided by David Grier, NYU-MRSEC and the Johns Hopkins Center for Talented Youth); C-blue) feedback collected from teachers $(\mathrm{n}=35)$ from Math for America: on two successive years, the teachers took a professional development mini-course at BioBus community laboratories, called "Demystifying Microscopes", learning to use the DIY optical kit. Teachers were encouraged to bring their acquired knowledge from these mini courses into their classrooms in NYC public schools. (Data kindly provided by John Russell, Math for America)

\subsubsection{DIY Fluorescence microscope}

Fluorescence microscopy is the technique of choice in many science laboratories to image biological preparations. Labelled with fluorescent indicators, biological structures can be visualized over the homogeneous background with high contrast. Traditionally, fluorescent labelling required chemical stains, most of which are lethal to biological samples ${ }^{9}$. In more recent years, the discovery of Green Fluorescent Protein (GFP) ${ }^{10}$ enabled scientists to perform fluorescent imaging in living samples, and even entire organisms ${ }^{11}$ through genetic engineering and other gene targeting approaches 12. GFP was further modified to obtain fluorescent markers of different colors ${ }^{13}$, as well as functional markers sensitive to transient cellular conditions, such as rising calcium waves ${ }^{14,15,16}$ or voltage differences across the cell membrane ${ }^{17,18}$. A newly introduced technique allowing tracking individual fluorescent molecules was recognized with the 2014 Nobel Prize in Chemistry. Unfortunately, despite the fact that fluorescent microscopes are widely used in research laboratories, their elevated cost prevents their use in most schools and educational institutions.

We modified the basic structure of the DIY compound microscope described above for trans-fluorescent imaging (Figure 4A). The white LED (Figure 1C) was replaced with a blue LED (450-460 nm) for sample illumination. 
Additionally, a long-pass green filter $(520 \mathrm{~nm})$ was introduced in front of the camera. We used the DIY fluorescent microscope to image preparations of larval fruit fly (D. melanogaster) dissected along the medial line to expose the brain and mouth hooks (Figure 4B). This transgenic organism expressed GFP in its neurons, permitting detailed imaging of its nervous system. The images in figure 4C-D show the two lobes of the central brain. Figure 4C was captured using the low-cost Raspberry Pi camera while figure 4D was captured using a Samsung Galaxy S6 camera. The fine nerve fibers emerging from the central brain $(5-10 \mu \mathrm{m})$ are visible in both images. Although the contrast is higher in the Raspberry Pi camera image (Figure 4C), the use of the phone camera has an advantage in terms of diffusion of the device (almost all students have a phone), as well as outreach and communication purposes. For example, phone images can be instantaneously shared through social media.

A

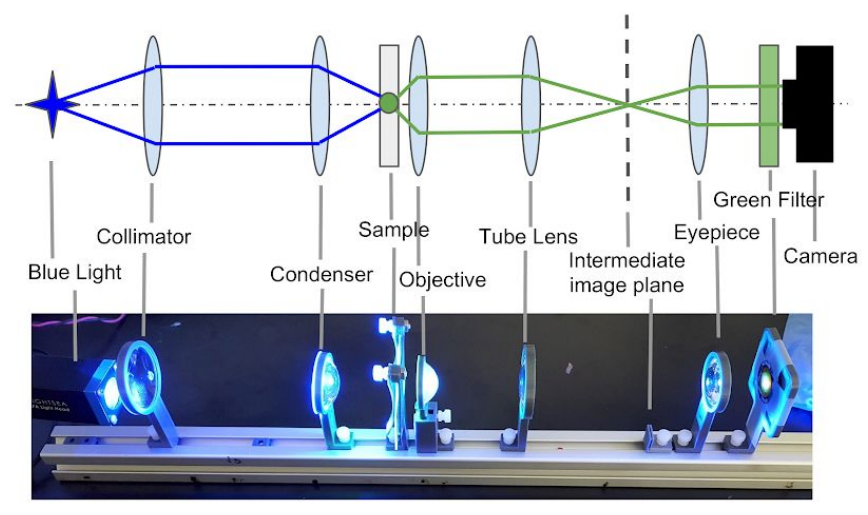

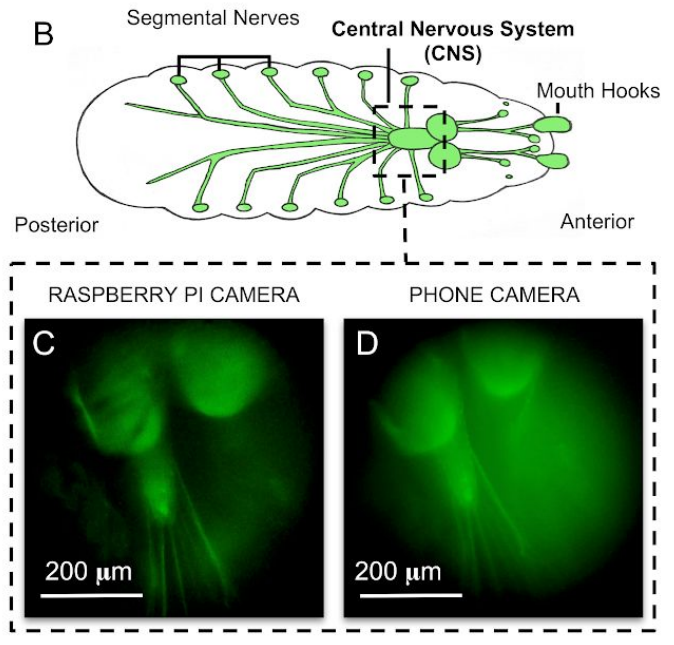

Figure 4: DIY fluorescent microscopy A) diagram of the optical configuration used for fluorescence imaging and photo of the DIY microscope configured accordingly; B) diagram of larval drosophila nervous system, with the central nervous system (CNS) highlighted; C) fluorescence images of larval drosophila CNS: left captured using Raspberry Pi Camera, right captured using cell phone camera (Samsung Galaxy S6).

\subsubsection{DIY infrared microscope for correction of chromatic aberration}

One of the disadvantages of white light imaging is blurring and distortion of the image due to chromatic aberrations ${ }^{19}$. This is caused by the unequal bending of the different wavelengths composing white light by lenses (Figure 5A). One method for avoiding this problem is to illuminate the sample with a narrower range of wavelengths.

With the DIY optical kit assembled in a compound microscope configuration, we substituted the white LED with an IR one $(850 \mathrm{~nm})$. Because IR light is not visible to the human eye, samples can only be viewed through a camera when illuminated in this way. Most commercial cameras have an integrated IR-blocking filter. However, cameras intended for use at night or in low-light conditions use IR light and so do not have an IR filter. We used one of these cameras, the low-cost Raspberry Pi Noir camera, as our detector. The DIY microscope so modified was used to demonstrate the benefits of narrow spectrum illumination for high-resolution imaging.

The results are shown in Figure 5B and E, where the same objects are imaged with broadband white light illumination (left) and narrow-spectrum IR illumination (right). Despite the fact that the low-cost plano-convex lenses and the aspherical objective lens used for the DIY microscope had no correction for chromatic aberration, the narrower illumination spectrum made it possible to capture sharper images at high magnification. Images obtained with white light illumination showed colored halos around objects, hallmarks of chromatic aberration. Replacing the illuminator with a narrow spectrum IR source eliminated chromatic aberration, allowing for sharply resolved images. Most notably, individual cells in the Elodea leaf could be seen clearly, as well as granules and other structures within the cells (Figure $\mathbf{5 E}$, right). Similarly, individual dendrites $(1-10 \boldsymbol{\mu m})$ were clearly resolved in the infrared IR image of mouse neurons 
(Figure 5B, right and Figure 5C), and single ommatidia were visible in the eye of the freshwater crustacean Daphnia magna (Figure 4B, right).
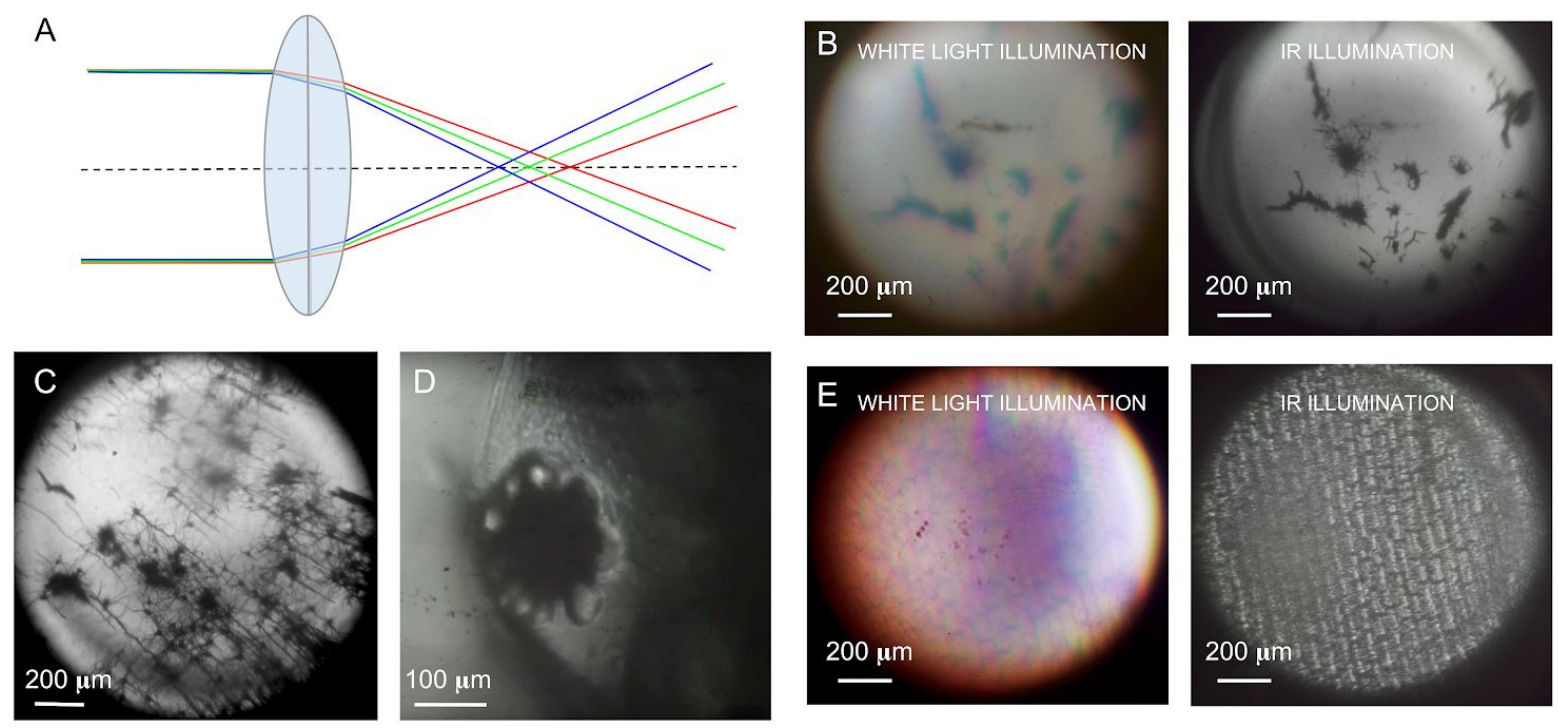

Figure 5: Infrared DIY microscope A) Diagram explaining chromatic aberration: parallel rays of different wavelengths (colors) are focused by a convex lens into slightly displaced points along the optical axis, deteriorating the resolution of the image and creating the characteristic "rainbow halo"; B) images of Golgi-stained mouse neurons captured using the DIY microscope: left using broadband white light illumination, right using IR illumination; C) mouse cortical neurons on a Golgi-stained brain slice imaged with the DIY IR microscope; D) compound eye of the freshwater crustacean Daphnia magna imaged with the DIY IR microscope; E) images of the aquatic plant Elodea: left using broadband white light illumination, right using IR illumination.

\subsubsection{Functional model of the human eye}

We used the DIY optical kit to build a functional model of the human eye. The structure of the eye allows for a complex system of functions to coexist and create the images that we see ${ }^{20}$. Optically, however, it can be simplified and represented by a combination of two lenses, the cornea and the crystalline lens, forming an image in the retina ${ }^{21}$, the neuronal tissue at the back of the eye that works as our "natural light sensor" (Figure 6A). The cornea allows for most of the focusing power, while the crystalline lens provides fine focus adjustments and forms the final image on the retina. Additionally, the crystalline lens confers the power of forming images of objects at different distances ${ }^{20}$. Here we show how the DIY optical kit can be used to demonstrate these fundamental properties of the human eye.

We modeled the basic structure of an eye using two lenses, corresponding to the cornea and crystalline lens, forming the image of an object on a paper screen representing the retina (Figure 6B). In our model, the cornea and crystalline lens remained stationary at a distance of $20 \mathrm{~mm}$ from each other, and $95 \mathrm{~mm}$ from the retina, mimicking the structure of a human eye where distances are determined by the anatomy and do not change. We began by modelling the cornea of a healthy eye with a convex lens of $70 \mathrm{~mm}$ focal length (FL). We then mimicked the crystalline lens using a second convex lens of $\mathrm{FL}=400 \mathrm{~mm}$. The object itself was a drawing painted on a microscope slide positioned $100 \mathrm{~mm}$ away from the cornea, and illuminated by a LED light source (Figure 6B). The image was focused by the two lenses onto the screen/retina (Figure 6C). Note that, as in a real eye, the image on the retina was upside down.

Our eye is able to form images of objects both closeby and far away. This is made possible by the ciliary muscles, that stretch and compress the crystalline lens thus modifying its effective focal length. This adjustment allows the images of objects at different distances to be focused in the retina. In our model, we mimicked the action of the ciliary muscles compressing the crystalline lens to focus on closeby objects by substituting the $400 \mathrm{~mm}$ FL crystalline lens (Figure 6C) 
with a thicker one, of $70 \mathrm{~mm}$ FL (Figure 6D). As expected, the object in focus on the retina was now closer to the "eye" ( $42 \mathrm{~mm}$ from the cornea $v s .100 \mathrm{~mm}$ in the faraway configuration). The size of the image on the retina also increased by a factor of 2, as it occurs in reality, where nearby objects appear larger than faraway ones.

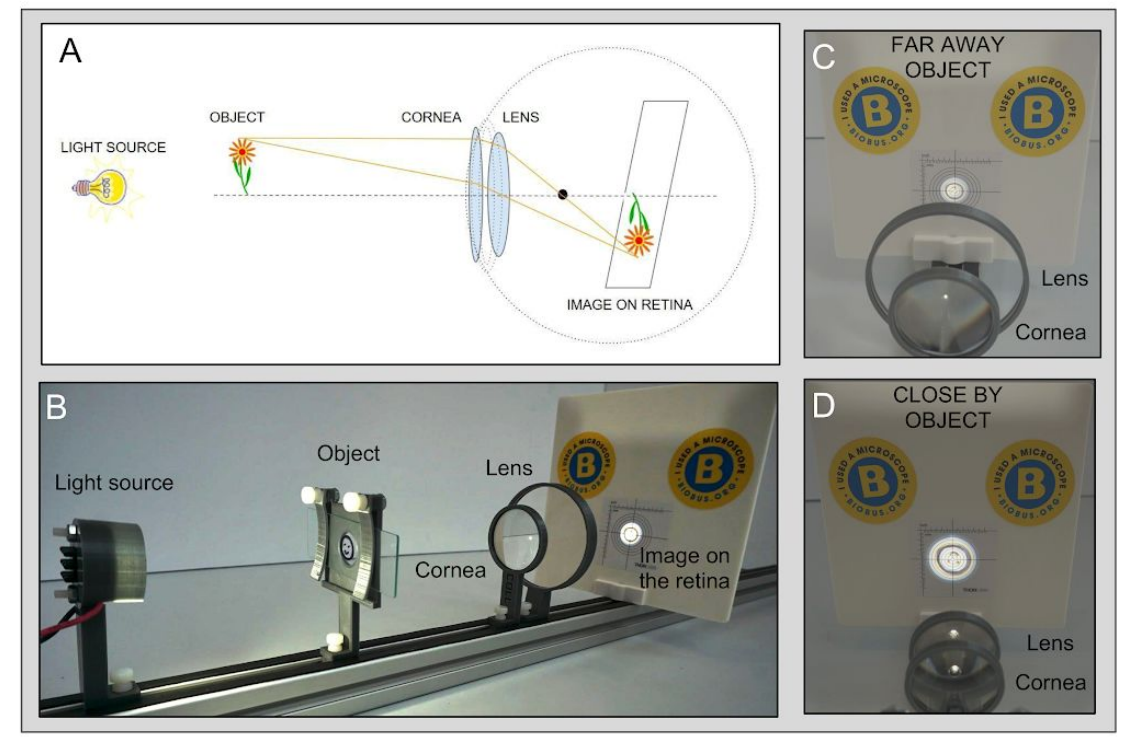

Figure 6: DIY functional model of the human eye. A) The main optical components of the eye are illustrated: cornea, lens and retina. Note that the image of the object on the retina is inverted, according to the rules of image formation. B) A normal functioning eye focusing an object at a distance modeled with the DIY optical kit. From left to right: LED light source, object (a face drawn on a microscope slide), cornea lens $(\mathrm{FL}=70 \mathrm{~mm})$, crystalline lens $(\mathrm{FL}=400 \mathrm{~mm})$, and retina (white screen). C) Front view of the DIY optical kit aligned to model a normal eye focusing a faraway object on the retina. The crystalline lens is modeled with a FL $=400 \mathrm{~mm}$ lens. D) Front view of the DIY optical kit aligned to model a normal eye focusing a closeby object on the retina. The crystalline lens is modeled with a $\mathrm{FL}=70 \mathrm{~mm}$ lens.

\subsubsection{Near and Farsightedness}

Common eye defects such as myopia (nearsightedness) and presbyopia (age-related farsightedness), can also be easily modeled with the DIY microscope. Myopia often occurs when the eyeball is too long, causing the image of faraway objects to be formed in front of the retina. As a result, faraway objects appear blurry, contrary to closeby ones that appear sharp. Myopia affects around 30\% of the U.S. population (American Optometric Association, 2018), and can be corrected by the use of concave glasses. To model this eye defect, we first aligned the DIY eye model in the "faraway" configuration, as described above (Figure 6B-C). We then moved the screen/retina backward by an additional $55 \mathrm{~mm}$, from the original configuration, to mimic a longer eyeball (Figure 7A). As expected, the image on the screen/retina became blurry (Figure 7B). Because the crystalline lens is already in its most relaxed configuration (long FL) and cannot be extended further by the ciliary muscles, the eye is not able to naturally correct this defect. However, inserting a concave lens ( $\mathrm{FL}=-45 \mathrm{~mm}$ ) between the object and the cornea, mimicking a corrective glass or contact lens (Figure 7C), represtinated a sharp image in the screen/retina (Figure 7D).

Presbyopia occurs when the image of nearby objects is formed at the back of the retina because of the hardening of the crystalline lens, which makes it impossible for the ciliary muscles to compress it and reduce its effective focal length. Presbyopia is a natural consequence of aging of the eye, with symptoms appearing progressively in people over 35 years old (National Eye Institute, 2010). To model this eye defect, we first aligned the DIY microscope in a "closeby" configuration (Figure 6D). We then substituted the short FL crystalline lens $(F L=70 \mathrm{~mm})$ with a longer FL lens $(\mathrm{FL}=$ $400 \mathrm{~mm}$, Figure 7E). This caused the image on the screen/retina to become blurry (Figure 7F). The effect could be 
reversed by introducing a convex lens $(\mathrm{FL}=30 \mathrm{~mm})$ between the object and the cornea, mimicking reading glasses (Figure 7G-H).

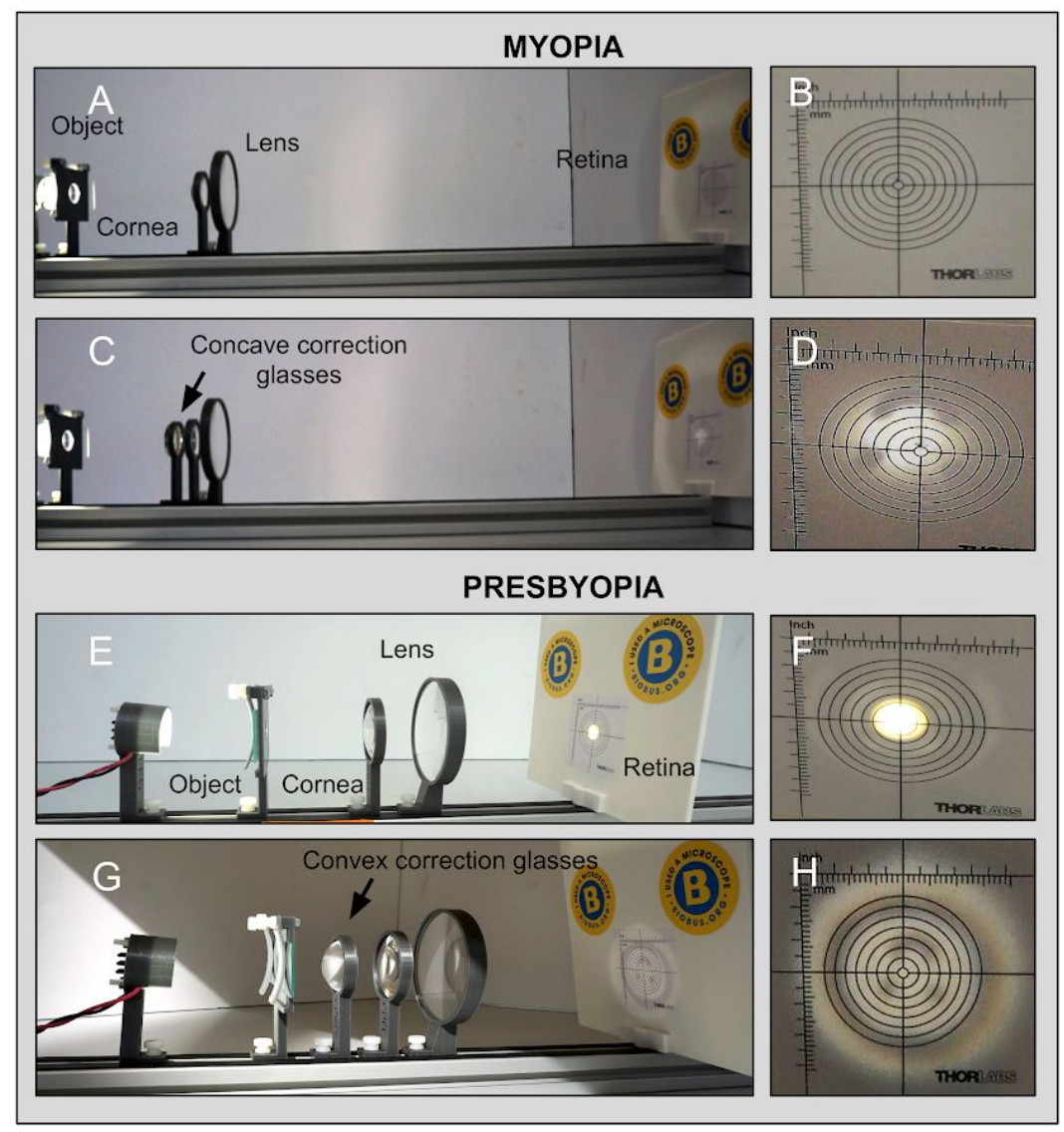

Figure 7: Eye defects. A) DIY optical kit arranged to model the eye of an individual affected by myopia, focusing on a faraway object. Note that the retina is farther away from the crystalline lens (150 mm vs. $95 \mathrm{~mm}$ in Figure 6B), to mimic the longer eyeball, typical of many forms of myopia. B) Front view of the screen/retina from A: as expected, there is no sharp image on the retina. C) DIY optical kit aligned as in A (myopia), but with the addition of a concave lens (FL $=-45 \mathrm{~mm}$ ) in front of the cornea to mimic correction glasses. D) Front view of the screen/retina from C: as expected, the image, an upside down smiley face, is back in focus on the retina. E) DIY optical kit arranged to model the eye of an individual affected by presbyopia, focusing on a closeby object. The crystalline lens is modeled by a lens with long $\mathrm{FL}=400 \mathrm{~mm}$ : this reflects the inability of the ciliary muscle to compress the hardened crystalline lens, and reduce its effective FL, typical of presbyopia. F) Front view of the screen/retina from E: as expected, there is no sharp image on the retina. G) DIY optical kit aligned as in E (presbyopia), but with the addition of a convex lens (FL $=30 \mathrm{~mm}) \mathrm{in}$ front of the cornea to mimic correction glasses. H) Front view of the screen/retina from G: as expected, the image, an upside down smiley face, is back in focus on the retina.

\subsubsection{Age-Related Macular Degeneration}

Subsequently, we used the DIY optical kit to model eye diseases. We chose to work on Age-Related Macular Degeneration (AMD), a debilitating disease affecting 1.75 million people in the U.S. ${ }^{22}$. Since AMD primarily occurs in people over 60 years of age, many of the elderly find themselves losing independence as their eyesight deteriorates. Through the progression of AMD, the central and most sensitive area of the retina, called the macula, is impeded in performing its functions ${ }^{23}$. There are two main forms of AMD, non-neovascular or dry (D-AMD) and neovascular or wet (W-AMD). In D-AMD, tissue debris, called drusen, accumulate in the macula until scattered blind spots occur in the central vision. In W-AMD, the formation of drusen further triggers the development of new blood vessels in the macula, causing blood leakage and resulting in even more pronounced blind spots ${ }^{24}$. 
We demonstrated the effects of AMD using our DIY model of the human eye. Drawing a black dot in the center of the screen/retina (macula), we mimicked the obstruction caused by drusen and growing blood vessels in a patient's central visual field (Figure 8 A-B). This is a very simple, yet optically accurate depiction of AMD, that we used for educational purposes during public science outreach events.

We then moved on to show how technology can be used to relieve the symptoms of AMD. In fact, although some drugs are currently available to reduce vasculature development in W-AMD ${ }^{25,26,27,28}$, there is no pharmacological treatment for D-AMD $28,29,23$. As an alternative, an Implantable Miniature Telescope (IMT) can be inserted into the eye, after removal of the crystalline lens, to recover partial vision ${ }^{30-32}$. When implanted in the eye, the IMT creates a "zoomed in" image of objects in the central visual field, thus reducing the relative size of the blind spots created by drusens, which are not magnified since they reside physically on the retina. The IMT is designed for use in only one eye because the increased size of the central visual field comes at the expense of peripheral vision. After appropriate physical therapy, however, the brain is able to use the implanted eye for central vision, and the other eye for peripheral vision, represtinating quasi-normal sight ${ }^{30}$.

We mimicked the action of the IMT by using a variation of our DIY eye model, where the IMT itself was modeled by a convex lens $(\mathrm{FL}=70 \mathrm{~mm})$ and concave lens $(\mathrm{FL}=-45 \mathrm{~mm})$ aligned to form a Galilean telescope (inter-lens distance 35 $\mathrm{mm}$, equal to the sum of the focal lengths, Figure 8C). During surgery, the crystalline lens is removed and the IMT is implanted in its place. Accordingly, in our model, the crystalline lens was removed, and the telescope was added between the cornea and the screen/retina (Figure 8C). Since the original IMT was designed to allow perfect vision of faraway objects, we aligned the DIY microscope as in Figure 6B (faraway configuration). As expected, the image produced by the telescope on the retina was enlarged by 1.6 times (Figure 8D). This is in agreement with what happens in real life, where the insertion of the IMT would allow patients to see a magnified version of objects in their central visual field. As in real life, our model showed that the relative size of the blind spot was reduced in the final, enlarged image (Figure 8D).

Patients implanted with the IMT lose their natural ability to focus on closeby objects, since the crystalline lens is removed. They correct the problem by using reading glasses. As expected, in our model, if the object was placed in the closeby configuration ( $42 \mathrm{~mm}$ from the cornea), no sharp image could be formed in the retina (Figure 8 E-F). The use of a convex lens $(\mathrm{FL}=30 \mathrm{~mm})$ between the object and the cornea to mimic reading glasses repestinated normal vision (Figure 8 G-H). Note that once again, the image of closeby objects (Figure 8H) is larger than that of faraway objects (Figure 8D). In AMD patients, this further reduces the relative size of the drusen.

\section{DISCUSSION}

In recent years, the development of low cost optics and 3D printing technology has allowed the production of cost-efficient Do-It-Yourself (DIY) microscopes, making these powerful scientific tools widely available to students, schools, and science enthusiasts around the world. One remarkable example is the Foldscope, a fully functional cardboard microscope that achieves micrometric resolution for less than a dollar production cost ${ }^{33}$. Other examples of DIY microscopes include the Smartphone Microscope by Infinite Scope and the Community Microscope kit from Public Lab. Other organizations have been promoting research-grade, low-cost optical technology for laboratories, as the "\$100 lab" by Open Neuroscience, an integrated optical platform for optogenetics and behavioral experiments ${ }^{34}$.

In this exciting scenario, the BioBus DIY optical kit responds to a different need, being at the same time a low cost microscope powerful enough to visualize single cells (micrometric resolution) and a pedagogical tool to teach the 


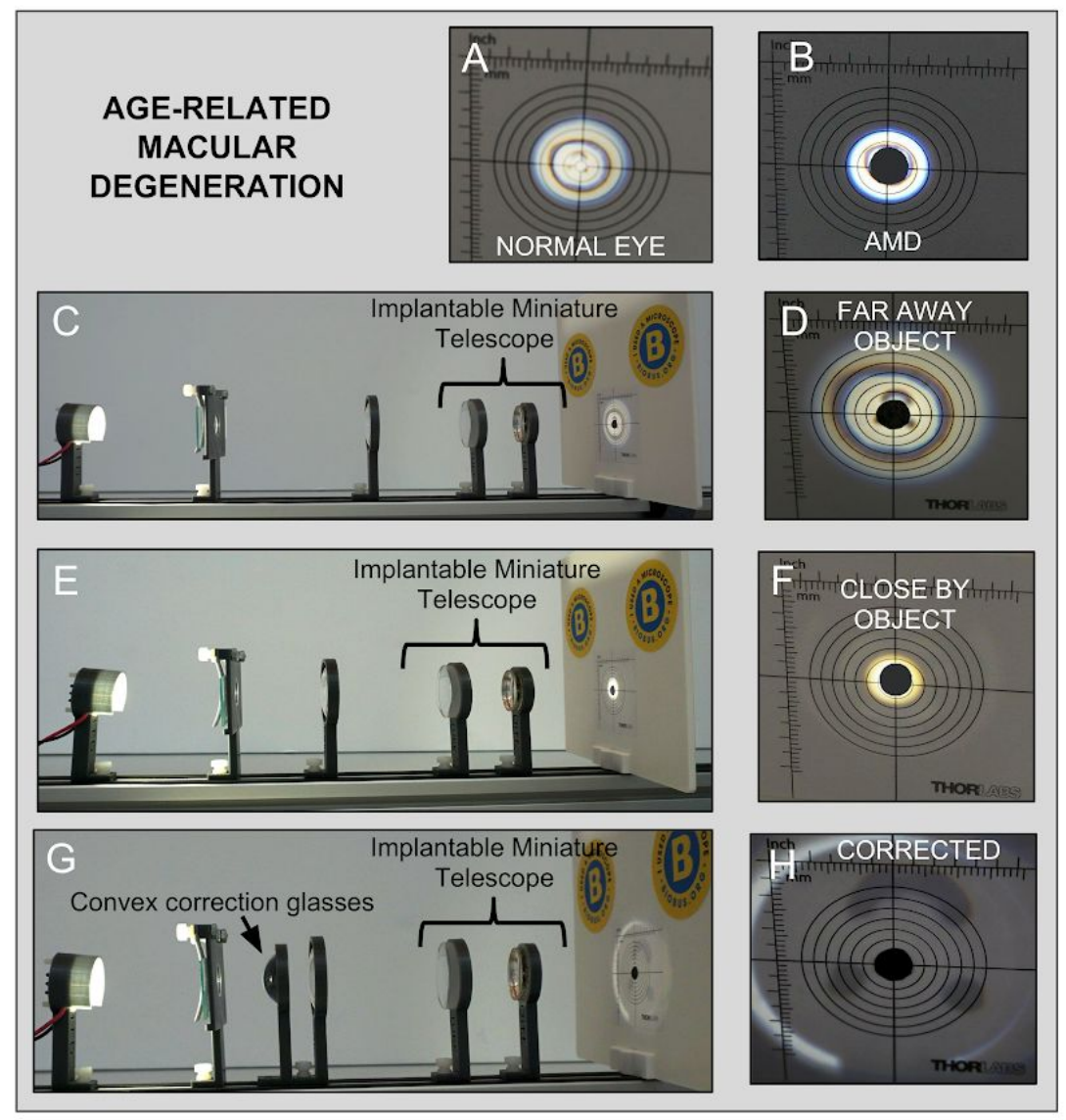

Figure 8: Age-related Macular Degeneration and the Implantable Miniature Telescope. A) Image of the object (smiley face painted on a microscopy slide) as formed by a "normal eye" aligned as in Figure 6B. B) Same object imaged by the eye of a patient affected by AMD: the drusen/outgrown vasculature are modeled by a black spot in the center of the screen/retina. Notice how the central visual field is completely obstructed. C) Model of an eye with Implantable Miniature Telescope (IMT): the crystalline lens was removed, as in real-life surgery. The IMT was modeled by a convex lens $(\mathrm{FL}=70 \mathrm{~mm})$ and a concave lens $(\mathrm{FL}=-45 \mathrm{~mm})$, aligned in a Galilean telescope configuration (inter-lens distance $35 \mathrm{~mm}$ ). D) Front view of the screen/retina from E: the image on the retina is enlarged by 1.6 times compared to B. As a result, the relative size of the drusen (black spot) is decreased, allowing for partial recovery of central vision. E) Model eye with IMT aligned as in C, but object in closeby configuration (42 $\mathrm{mm}$ from cornea). F) Front view of the screen/retina from E: as expected, there is no sharp image on the retina. G) Model eye with IMT aligned as in E (closeby configuration), but with the addition of a convex lens $(\mathrm{FL}=30 \mathrm{~mm})$ in front of the cornea to mimic correction glasses. $\mathrm{H})$ Front view of the screen/retina from $\mathrm{G}$ : as expected the enlarged image is back in focus on the retina.

fundamentals of optics and microscope design. We showed that the modular, easy-to-use design of our optical kit is able to inspire creativity and ignite interest in students as young as eight years-old, allowing them to create and assemble their own microscopes, an experience normally reserved for graduate students in advanced microscopy laboratories. The use of 3D printing technology and surplus optical supplies allowed us to limit the production cost of the BioBus DIY microscope to less than $\$ 200$, an order of magnitude cheaper than comparable optical kits assembled from commercial components ${ }^{35}$. This in turn ensures that our product is affordable enough to be deployed in science classrooms around the country, and especially in historically underserved communities where BioBus, Inc. concentrates its action.

We took advantage of the expertise in science outreach and education acquired by BioBus over the years to develop two guided-inquiry curricula to support educators in the use of our DIY optical kit. The curricula were further refined thanks to the feedback from educators and scientists from our NYC partners, Math for America and NYU-MRSEC. These curricula are available upon request. We are currently fundraising to develop a dedicated website that would allow us to 
make the DIY microscopes design files and curricula widely available to the national and international public. In this endeavor, we are encouraged by the feedback collected so far, indicating that our DIY optical kit has the potential to make optics interesting and accessible to a large range of school-aged students and their teachers.

As we showed in the second part of this manuscript, the DIY optical kit can also be used as a platform for the development of advanced microscopy projects. These projects have been conceived and directed by high school and college students, working as interns at BioBus, under the supervision of our resident scientists. The results obtained by our students offer another demonstration that $3 \mathrm{D}$ printing technology is now mature enough to support the production of research-quality optics at reduced costs. This adds to the recent trend of engineering developments furthering the democratization of science technology and education. These developments do not just benefit the fields of optics and physics, but can be the catalyst for fruitful interdisciplinary cross-pollination, as we showed here by transforming our DIY optical kit into a functional model of the human eye. This model was used to illustrate the complexity of eye physiology to students, in a visual and relatable way. It also contributed to raise public awareness and understanding of common eye defects and diseases, and to enhance the appreciation of the importance of optical physics in everyday life.

BioBus works towards a future where every person has the opportunity to reach their full scientific potential. We will continue to work on the DIY microscope project, investing in its dissemination to the wider public through outreach initiatives and the creation of a dedicated website. We will also continue to support our students in their pursuit of advanced optical and interdisciplinary projects. We hope, in this way, to make a potentially far-reaching contribution to promote talent and help shape a more diverse future generation of scientists and engineers.

\section{ACKNOWLEDGEMENTS}

This project is not just the work of a few individuals, but rather the result of the engagement of an entire community. As such, we would like to thank first and foremost all the members of the BioBus community, especially our students who make all of this worthwhile. Special thanks to the scientific and support staff from BioBus Inc., in particular Benjamin Dubin-Thaler, Robert Frawley, Sarah Weisberg, and Tiffany King for their support of the development of the project, Benjamin Dubin-Thaler, Sasha Chait, Roya Heydari and Victoria Von Ancken for help with the manuscript revision. We warmly thank our collaborators from New York University's Material Research Science and Engineering Center, Math for America, Regeneron Pharmaceuticals and the Jingtai Cao laboratory, the Washington Heights Expeditionary Learning School, the Johns Hopkins Center for Talented Youth and World Science Festival. We are grateful to David Grier for helpful conversations. We would like to thank Jane Rhee, Katie Lowen, Michael Seto, Matthew and Kathy Koh, Darcy Peterka, Aurelien Begue, James Scholz, Neli Prota and Dario Bressan for help and inspiration; Natalie Kolba, Priyanka Gupta and Elizabeth Waters for providing exciting samples.

Funding for this project was provided by Math for America, the Johns Hopkins Center for Talented Youth, Science Sandbox (an initiative of the Simons Foundation), Regeneron Pharmaceuticals, and donations from individuals. Partial support was provided by the MRSEC Program of the National Science Foundation through Award Number DMR-1420073. M.F.S. participation in the conference was sponsored by Darcy Peterka's lab at Columbia University's Zuckerman Institute.

\section{REFERENCES}

[1] "Milestones in light microscopy, timeline.", Nature (2009).

[2] Bozzola, J. J. and Russell, L. D., [Electron Microscopy: Principles and Techniques for Biologists], Jones \& Bartlett Learning, Sudbury MA, 2-16 (1999).

[3] Cheng, P.-C. and Jan, G.-J., [X-ray Microscopy: Instrumentation and Biological Applications], Springer Science \& Business Media, Berlin Heidelberg, 1-12 (2012).

[4] Muller, M., [Introduction to Confocal Fluorescence Microscopy], SPIE Press, Bellingham WA, 1-23 
(2006).

[5] Denk, W., Strickler, J. H. and Webb, W. W., "Two-photon laser scanning fluorescence microscopy," Science 248(4951), 73-76 (1990).

[6] Stohr-Hunt, P. M., "An analysis of frequency of hands-on experience and science achievement," Journal of Research in Science Teaching 33(1), 101-109 (1996).

[7] Howe, L., "An Exploratory Case Study Of Upper Elementary And Middle Level Science Instruction Impacting Traditionally Underserved Students," Ph.D., K. Seide, Ed. (2012).

[8] C., V. S., "Science achievement in social contexts: Analysis from national assessment of educational progress," The Journal of Educational Research 98(2), 67-78 (2004, Nov 1).

[9] Renz, M., "Fluorescence microscopy-A historical and technical perspective," Cytometry A 83(9), 767-779 (2013).

[10] Shimomura, O., Johnson, F. H. and Saiga, Y., "Extraction, purification and properties of aequorin, a bioluminescent protein from the luminous hydromedusan, Aequorea," J. Cell. Comp. Physiol. 59, 223-239 (1962).

[11] Chalfie, M., Tu, Y., Euskirchen, G., Ward, W. W. and Prasher, D. C., "Green fluorescent protein as a marker for gene expression," Science 263(5148), 802-805 (1994).

[12] Schneider, A. F. L. and Hackenberger, C. P. R., "Fluorescent labelling in living cells," Curr. Opin. Biotechnol. 48, 61-68 (2017).

[13] Palmer, A. E., Qin, Y., Park, J. G. and McCombs, J. E., "Design and application of genetically encoded biosensors," Trends Biotechnol. 29(3), 144-152 (2011).

[14] Miyawaki, A., Llopis, J., Heim, R., McCaffery, J. M., Adams, J. A., Ikura, M. and Tsien, R. Y., "Fluorescent indicators for $\mathrm{Ca} 2+$ based on green fluorescent proteins and calmodulin," Nature 388(6645), 882-887 (1997).

[15] Romoser, V. A., Hinkle, P. M. and Persechini, A., "Detection in living cells of Ca2+-dependent changes in the fluorescence emission of an indicator composed of two green fluorescent protein variants linked by a calmodulin-binding sequence. A new class of fluorescent indicators," J. Biol. Chem. 272(20), 13270-13274 (1997).

[16] Persechini, A., Lynch, J. A. and Romoser, V. A., "Novel fluorescent indicator proteins for monitoring free intracellular Ca2," Cell Calcium 22(3), 209-216 (1997).

[17] Siegel, M. S. and Isacoff, E. Y., "Green fluorescent proteins (GFPs) for measuring voltage," Cold Spring Harb. Protoc. 2010(4), db.top76 (2010).

[18] Baker, B. J., Mutoh, H., Dimitrov, D., Akemann, W., Perron, A., Iwamoto, Y., Jin, L., Cohen, L. B., Isacoff, E. Y., Pieribone, V. A., Hughes, T. and Knöpfel, T., "Genetically encoded fluorescent sensors of membrane potential," Brain Cell Biol. 36(1-4), 53-67 (2008).

[19] Murphy, D. B., [Fundamentals of Light Microscopy and Electronic Imaging], John Wiley \& Sons, New York NY, 43-60 (2002).

[20] Hubel, D. H., [Eye, Brain, and Vision], W. H. Freeman, New York NY (1995).

[21] Atchison, D. A. and Smith, G., [Optics of the Human Eye], Butterworth-Heinemann Medical, Woburn MA, 3-10 (2000).

[22] "Prevalence of Age-Related Macular Degeneration in the United States.", Arch. Ophthal. 122(4), 564 (2004).

[23] Ambati, J. and Fowler, B. J., "Mechanisms of age-related macular degeneration,” Neuron 75(1), 26-39 (2012).

[24] Bressler, N. M., Bressler, S. B. and Fine, S. L., "Age-related macular degeneration,” Surv. Ophthalmol. 32(6), 375-413 (1988).

[25] Heier, J. S., Brown, D. M., Chong, V., Korobelnik, J.-F., Kaiser, P. K., Nguyen, Q. D., Kirchhof, B., Ho, 
A., Ogura, Y., Yancopoulos, G. D., Stahl, N., Vitti, R., Berliner, A. J., Soo, Y., Anderesi, M., Groetzbach, G., Sommerauer, B., Sandbrink, R., Simader, C., et al., "Intravitreal Aflibercept (VEGF Trap-Eye) in Wet Age-related Macular Degeneration," Ophthalmology 119(12), 2537-2548 (2012).

[26] Schmidt-Erfurth, U., Kaiser, P. K., Korobelnik, J.-F., Brown, D. M., Chong, V., Nguyen, Q. D., Ho, A. C., Ogura, Y., Simader, C., Jaffe, G. J., Slakter, J. S., Yancopoulos, G. D., Stahl, N., Vitti, R., Berliner, A. J., Soo, Y., Anderesi, M., Sowade, O., Zeitz, O., et al., "Intravitreal aflibercept injection for neovascular age-related macular degeneration: ninety-six-week results of the VIEW studies," Ophthalmology 121(1), 193-201 (2014).

[27] Singer, M. A., Awh, C. C., Sadda, S., Freeman, W. R., Antoszyk, A. N., Wong, P. and Tuomi, L., "HORIZON: an open-label extension trial of ranibizumab for choroidal neovascularization secondary to age-related macular degeneration," Ophthalmology 119(6), 1175-1183 (2012).

[28] Eleftheriadou, M., Gemenetzi, M., Lukic, M., Sivaprasad, S., Hykin, P. G., Hamilton, R. D., Rajendram, R., Tufail, A. and Patel, P. J., "Three-Year Outcomes of Aflibercept Treatment for Neovascular Age-Related Macular Degeneration: Evidence from a Clinical Setting," Ophthalmol Ther (2018).

[29] "Website.", <Segato, T., Midena, E. \& Blarzino, M.C. Aging Clin Exp Res (1993) 5: 165. https://doi.org/10.1007/BF03324151> (21 July 2018 ).

[30] Lane, S. S., Kuppermann, B. D., Fine, I. H., Hamill, M. B., Gordon, J. F., Chuck, R. S., Hoffman, R. S., Packer, M. and Koch, D. D., "A prospective multicenter clinical trial to evaluate the safety and effectiveness of the implantable miniature telescope," Am. J. Ophthalmol. 137(6), 993-1001 (2004).

[31] Lipshitz, I., Loewenstein, A., Reingewirtz, M. and Lazar, M., "An intraocular telescopic lens for macular degeneration," Ophthalmic Surg. Lasers 28(6), 513-517 (1997).

[32] Agarwal, A., Lipshitz, I., Jacob, S., Lamba, M., Tiwari, R., Kumar, D. A. and Agarwal, A., "Mirror telescopic intraocular lens for age-related macular degeneration: design and preliminary clinical results of the Lipshitz macular implant,” J. Cataract Refract. Surg. 34(1), 87-94 (2008).

[33] Cybulski, J. S., Clements, J. and Prakash, M., "Foldscope: origami-based paper microscope," PLoS One 9(6), e98781 (2014).

[34] Maia Chagas, A., Prieto-Godino, L. L., Arrenberg, A. B. and Baden, T., "The $€ 100$ lab: A 3D-printable open-source platform for fluorescence microscopy, optogenetics, and accurate temperature control during behaviour of zebrafish, Drosophila, and Caenorhabditis elegans," PLoS Biol. 15(7), e2002702 (2017).

[35] Rachel Kemp Alexander Chippendale, Monica Harrelson, Jennifer Shumway, Amanda Tan, Sarah Zuraw, Jennifer L. Ross., "Microscope Project for Undergraduate Laboratories" (2016). 


\section{SUPPLEMENTARY MATERIAL}

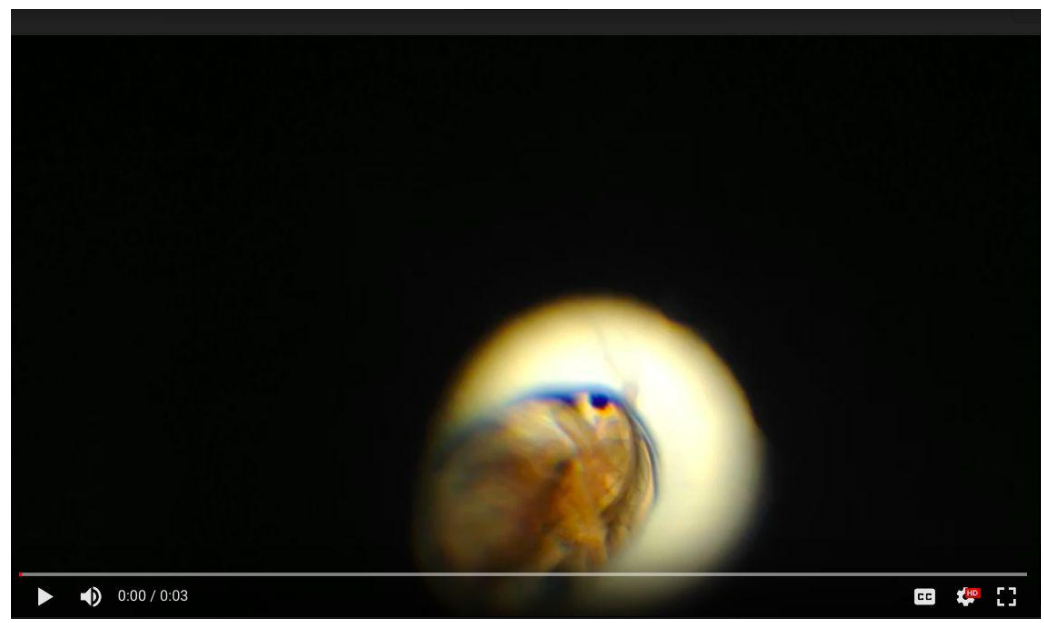

Video 1: Freshwater crustacean Daphnia magna imaged on a phone camera with the DIY optical kit aligned to form a compound microscope.

http://dx.doi.org/10.1117/12.2320655.1 